\title{
Well-width dependence of coupled Bloch-phonon oscillations in biased InGaAs/InAlAs superlattices
}

\author{
Michael Först*1, Heinrich Kurz ${ }^{1}$, Thomas Dekorsy ${ }^{2}$, and Richard P. Leavitt ${ }^{3}$ \\ ${ }^{1}$ Institut für Halbleitertechnik, RWTH Aachen University, Sommerfeldstrasse 24, 52074 Aachen, Germany \\ ${ }^{2}$ Institut für Ionenstrahlphysik und Materialforschung, Forschungszentrum Rossendorf, 031414 Dresden, \\ Germany \\ ${ }^{3}$ U.S. Army Research Laboratory, 2800 Powder Mill Road, Adelphi, Maryland 20783
}

\begin{abstract}
The coupling between Bloch oscillations and longitudinal optical (LO) phonons is investigated in ternary $\mathrm{In}_{0.53} \mathrm{Ga}_{0.47} \mathrm{As} / \mathrm{In}_{0.52} \mathrm{Al}_{0.48}$ As superlattices of different well widths. In femtosecond time-resolved studies, a strong increase of the coherent LO phonon amplitudes is observed when the Bloch oscillations are subsequently tuned into resonance with the different optical phonon modes of the ternary semiconductor materials. In a narrow-well superlattice where electronic minibands are energetically shifted close to the confining barrier potential, the phonon amplitudes are asymmetrically enhanced on the high-frequency edge of the resonance. Here, at high electric fields, field induced tunneling into above-barrier continuum states leads to a rapid dephasing of Bloch oscillations. The associated polarization change provides an additional excitation process for coherent LO phonons.
\end{abstract}

1 Introduction Time-resolved investigations of resonantly coupled excitations involving phonons, such as coupled plasmon-phonon modes or phonon-polaritons, are one of the most intriguing subjects of coherent phonon spectroscopy (for a review see, e.g., [1]).

Besides the elementary excitations involved there (plasmons, light waves), the coherent excitation of Bloch oscillations (BO's) has become possible in semiconductor superlattices by use of femtosecond laser pulses (for a review see, e.g., [2]). In general, BO's are described as the spatial oscillation of carriers in a periodic potential subject to a static electric field $[3,4]$. In biased superlattices, the coherent superposition of electronic Wannier-Stark (WS) states by ultrashort laser pulses leads to the formation of an electronic wave packet oscillating with a frequency $\nu_{B O}=e F d / h$. This frequency is determined by the externally applied electric field $F$, the superlattice period $d$, the electron charge $e$, and Planck's constant $h$.

Recently, the resonant coupling between optical phonons and BO's has been demonstrated for the first time in GaAs/AlGaAs superlattices [5]. Here, we present a study on coupled Bloch-phonon oscillations in $\mathrm{In}_{0.53} \mathrm{Ga}_{0.47} \mathrm{As} / \mathrm{In}_{0.52} \mathrm{Al}_{0.48} \mathrm{As}$ superlattices of different well widths but constant electronic miniband widths. A strong increase of the coherent LO phonon amplitudes is observed when the BO's are subsequently tuned into resonance with the distinct phonon modes of the ternary superlattice semiconductors by variation of the applied electric field. Furthermore, narrow superlattice wells lead to a shift of electronic minibands to higher energies in the conduction band and, thus, to an enhancement of Zener tunneling into above-barrier continuum states at high electric fields [6]. This effect results in a rapid dephasing of coherent BO's that in turn induces an incoherent polarization exciting coherent LO phonons. This contribution manifests itself as an asymmetric enhancement of the LO phonon amplitudes on the high-frequency edge of the Bloch-phonon resonance.

\footnotetext{
* Corresponding author: e-mail: foerst@ @iht.rwth-aachen.de, Phone: +49 24180 27896, Fax: +49 2418022246
} 
2 Experimental Two samples with different energetic positions of the first electronic minibands in the conduction band but constant miniband widths of $60 \mathrm{meV}$ were investigated in femtosecond time-resolved experiments. For the resonant excitation of BO's in sample A with well/barrier widths of 8.4/51.6 (energetically high miniband, i.e. close to the confining barrier potential), a Ti:sapphire laser delivering pulses of 45 fs lengths was tuned to a photon energy of $1.39 \mathrm{eV}$. For sample B with well/barrier widths of 35.4/24.6 $\AA$ (energetically low miniband, i.e. approximately $400 \mathrm{meV}$ beneath the confining barrier potential), we used a synchronously pumped optical parametric oscillator with photon energies of $1.00 \mathrm{eV}$ and pulse lengths of $70 \mathrm{fs}$.

The LO phonons of the ternary superlattice materials exhibit a typical two-mode behaviour. For bulk compounds grown lattice-matched to an InP substrate, their Brillouin zone center frequencies are $7.1 \mathrm{THz}$ for the InAs-like modes in $\operatorname{In}_{0.53} \mathrm{Ga}_{0.47} \mathrm{As}$ and $\mathrm{In}_{0.52} \mathrm{Al}_{0.48} \mathrm{As}\left(\mathrm{LO}_{I}\right), 8.0 \mathrm{THz}$ for the GaAs-like mode in $\mathrm{In}_{0.53} \mathrm{Ga}_{0.47} \mathrm{As}\left(\mathrm{LO}_{I I}\right)$, and $11.0 \mathrm{THz}$ for the AlAs-like mode in $\operatorname{In}_{0.52} \mathrm{Al}_{0.48} \mathrm{As}\left(\mathrm{LO}_{I I I}\right)[7,8]$.

The investigations were carried out in an electrooptic detection scheme (REOS, reflective electrooptic sampling) [9]. Here, polarization dynamics arising from optically excited coherent carrier and lattice dynamics in the superlattice growth direction are monitored by anisotropic changes of the sample reflectivity in the time domain. The measurements were performed at lattice temperatures of $10 \mathrm{~K}$. The optical excitation density was kept below $3 \times 10^{9}$ electron-hole pairs per well and $\mathrm{cm}^{2}$ in order to reduce carrier-carrier scattering which leads to an enhanced dephasing of BO's.

3 Results Figure 1(a) shows the coupled dynamics of BO's and LO phonons detected in sample A in the voltage range between $-1.6 \mathrm{~V}$ and $-8.8 \mathrm{~V}$, i.e., within the $\mathrm{WS}$ regime of the superlattice. The coherent carrier and phonon contributions can be clearly distinguished by their voltage dependence. The BO-induced component for time delays below $300 \mathrm{fs}$ is characterized by an increase of its frequency and a concomitant decrease of its amplitude with increasing reverse bias. On the other hand, the phononinduced component comprises a beating of long-lived oscillations that shows no discernable change of the contributing frequencies but a remarkable amplitude increase if the applied voltage is varied.
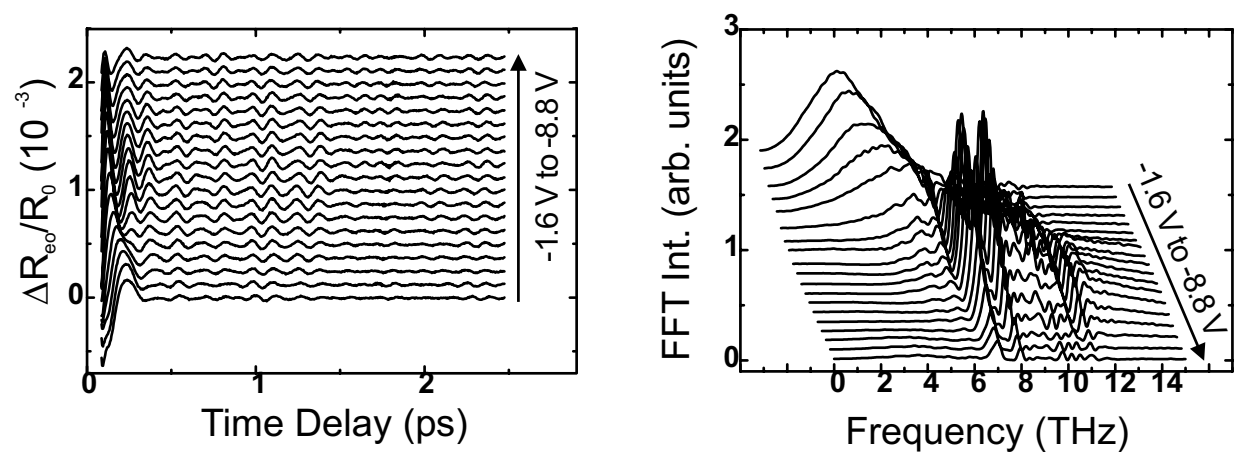

Fig. 1 (a) Numerically extracted oscillatory components of the REOS signals detected in sample A for reverse bias voltages between $-1.6 \mathrm{~V}$ and $-8.8 \mathrm{~V}$ (in steps of $-0.4 \mathrm{~V}$ ). (b) Fourier transforms of the data shown. For clarity, the traces and spectra are shifted vertically and horizontally.

The picture of coupled Bloch-phonon oscillations is confirmed by Fourier transforms of these data depicted in Fig. 1(b). In the low-voltage range, the Fourier transforms are dominated by spectrally broad peaks arising from BO's. As expected, their frequency increases linearly with increasing electric fields applied to the superlattice. A detailed analysis (not shown here, see [10] instead) yields a slope of $-1.4 \mathrm{THz} / \mathrm{V}$ in very good agreement with a theoretically expected value calculated from the superlattice parameters. The LO phonon modes of the superlattice appear as sharp peaks in the Fourier transforms 
at their eigenfrequencies listed above $\left(\mathrm{LO}_{I}-\mathrm{LO}_{I I I}\right)$. Obviously, when the $\mathrm{BO}$ frequency is tuned into resonance with the LO phonons a pronounced enhancement of the coherent phonon amplitudes is observed. This amplitude increase is attributed to the resonant coupling of the coherent electronic carrier motion, i.e. the BO's, to the phonon oscillations via their polarization components parallel to the growth direction of the superlattice [5]. Thus, BO's appear as an highly effective driving force for the generation of coherent phonons even at low excitation densities.
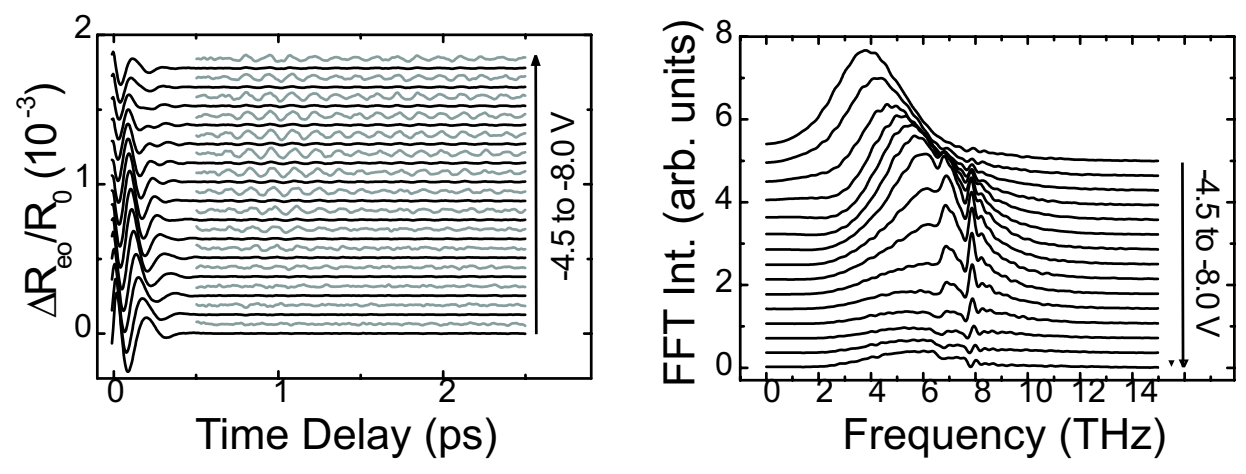

Fig. 2 (a) Oscillatory signal contributions recorded in sample B in the voltage range between $-4.5 \mathrm{~V}$ and $-8.0 \mathrm{~V}$ (in increments of $-0.25 \mathrm{~V}$ ). For clarity, the gray traces depict the long-living phonon oscillations enhanced by a factor of five. (b) Corresponding Fourier transforms of data shown in (a).

The main aspects derived from sample A are confirmed by experiments performed on sample B. Figure 2(a) depicts the coupled Bloch-phonon oscillations detected in sample B in the voltage range between $-4.5 \mathrm{~V}$ and $-8.0 \mathrm{~V}$ (for clarity, the gray traces depict the long-lived phonon oscillations enhanced by a factor of five). Again, BO-induced signal contributions can be distinguished from the long-lived phonon traces by the electric field dependence of their frequencies and amplitudes. The corresponding Fourier transforms shown in Fig. 2(b) manifest the resonant coupling between the coherent carrier and phonon dynamics. When the Bloch peak approaches the optical phonon frequencies, a pronounced enhancement of the coherent $\mathrm{LO}_{I}$ and $\mathrm{LO}_{I I}$ phonon amplitudes is observed at frequencies of 7.1 and $8.0 \mathrm{THz}$. Note that in contrast to the Ti:sapphire laser based experiments on sample A, longer pulses delivered by the optical parametric oscillator suppress the observation of the $\mathrm{LO}_{\text {III }}$ phonon at $11.0 \mathrm{THz}$.

For a more detailed analysis and a comparison of the resonant Bloch-phonon coupling for both samples, the coherent LO phonon amplitudes (normalized to their maximum value) are plotted versus the BO frequency in Fig. 3. For this purpose, the phonon amplitudes were determined from Fourier transforms for larger time delays only, thus omitting contributions of the rapidly dephasing BO's. The distinct peak positions of the LO phonons are determined from Gaussian shaped numerical fits to the rising edge of the data, additionally shown in Fig. 3. For both samples, the LO phonon amplitudes reach their maximum value at $\mathrm{BO}$ frequencies very close to the corresponding phonon eigenfrequencies. This selective enhancement, again, clearly demonstrates the resonant behaviour of the coupling between the frequency tunable BO's and the coherent LO phonons.

Nevertheless, the shape of the Bloch-phonon resonance coupling differs for the two samples investigated. In contrast to sample B, where the LO phonon amplitudes are symmetrically enhanced in the vicinity of the resonance, an asymmetry of the phonon amplitudes is observed as a function of the BO frequency in sample A. This discrepancy is attributed to the different energetic positions of the electronic minibands and the consequences for the dephasing of the coherent BO's.

In sample B, the BO's are excited in an electronic miniband far below (approximately $400 \mathrm{meV}$ ) the barrier potential of the conduction band. Here - neglecting resonant effects like, e.g., resonant phonon emission between WS states [11] - the dephasing is considered to be independent on the applied electric 


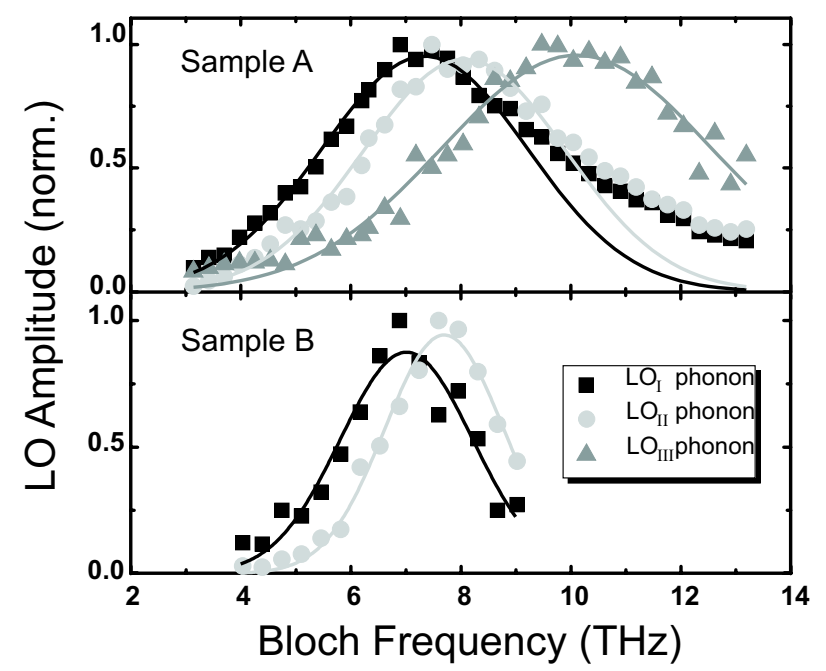

Fig. 3 Normalized amplitudes of the coherent LO phonons (see text) versus the BO frequency. The solid lines are Gaussian fits to the low-frequency edge of the resonance.

field and a symmetric Bloch-phonon resonance is expected [12]. In the narrow-well superlattice (sample A), however, BO's are excited in a shallow bound electronic miniband only $20 \mathrm{meV}$ (upper edge) below the potential barriers. Here, the rapid dephasing of BO's leads to an additional current arising from tunneling processes of the optically excited electrons into the above-barrier continuum states [6]. At high electric fields, the associated charge current is able to provide a bandwidth large enough for the coherent excitation of LO phonons. This excitation mechanism superimposed on the resonant Bloch-phonon coupling leads to an asymmetric increase of the coherent phonon amplitudes above the LO phonon frequencies compared to the amplitudes below the phonon resonances.

Acknowledgements The authors thank A. Bartels for valuable discussions and H. Giessen for providing the optical parametric oscillator. The Deutsche Forschungsgemeinschaft, the Ministerium für Schule, Wissenschaft und Forschung Nordrhein-Westfalen, and the European Research Office of the U.S. Army are acknowledged for financial support.

\section{References}

[1] T. Dekorsy, G. C. Cho, and H. Kurz, Coherent Phonons in Condensed Media, Light Scattering in Solids, Vol. VIII, eds. M. Cardona and G. Güntherodt (Springer Verlag, Berlin, 2000), p. 169.

[2] K. Leo, Semicond. Sci. Tech. 13, 249 (1998).

[3] F. Bloch, Z. Phys. 52, 555 (1928).

[4] C. Zener, The theory of the Electrical Breakdown of Solid Dielectrics, Proc. R. Soc. London A 145, 523 (1934).

[5] T. Dekorsy, A. Bartels, H. Kurz, K. Köhler, R. Hey, and K. Ploog, Phys. Rev. Lett. 85, 1080 (2000).

[6] B. Rosam, D. Meinhold, F. Löser, V. G. Lyssenko, S. Glutsch, F. Bechstedt, F. Rossi, K. Köhler, and K. Leo, Phys. Rev. Lett. 86, 1630 (2001).

[7] P. Bhattacharya (ed.), Properties of Lattice-matched and Strained InGaAs (INSPEC, London, 1993).

[8] L. Pavesi, R. Houdré, and P. Gianozzi, J. Appl. Phys. 78, 470 (1999).

[9] T. Dekorsy, P. Leisching, K. Köhler, and H. Kurz, Phys. Rev. B 50, 8106 (1994).

[10] M. Först, H. Kurz, T. Dekorsy, and R.P. Leavitt, Phys. Rev. B 67, 085305 (2003).

[11] J. Hader, T. Meier, S. W. Koch, F. Rossi, and N. Lindner, Phys. Rev. B 55, 13799 (1997).

[12] A. W. Ghosh, L. Jönsson, and J. W. Wilkins, Phys. Rev. Lett. 85, 1084 (2000). 